\title{
No-Go theorems and quantization
}

\author{
Hishamuddin Zainuddin, ${ }^{\text {a,b, }}{ }^{*}$ Toh Sing Poh, ${ }^{a}$ Nurisya Mohd Shah, ${ }^{\text {a,b }}$ Mazlinda Zainy, ${ }^{b}$ Zuriati Zulkarnain, ${ }^{\text {a,c }}$ \\ Jumiah Hassan $^{\mathrm{a}, \mathrm{b}}$ and Zainul Abidin Hassan ${ }^{\mathrm{a}, \mathrm{b}}$ \\ ${ }^{a}$ Theoretical Studies Laboratory, Institute for Mathematical Research and \\ ${ }^{b}$ Department of Physics, Faculty of Science Universiti Putra Malaysia, 43400 UPM Serdang, Selangor. \\ ${ }^{c}$ Department of Communications Technology \& Networking, Faculty of Computer Science \& Information Technology Universiti \\ Putra Malaysia, 43400 UPM Serdang, Selangor \\ *To whom correspondence should be addressed. E-mail: hisham@fsas.upm.edu.my
}

Received 10 November 2006

http://dx.doi.org/10.11113/mjfas.v3n1.21

\begin{abstract}
In this review, we would like to highlight the three known no-go theorems in quantum physics in relation to the process of quantization that maps classical observables to quantum ones. The quantization approach considered is a mixture of Isham's group-theoretic quantization and geometric quantization with special emphasis on underlying compact phase space geometry of spheres. The first is Groenewold-van Hove theorem that states the obstruction of quantizing the full algebra of observables and in the sphere case, only limited to the spin observables plus the constant functions. The other two are theorems of Bell and Kochen-Specker stating that the only hidden variable theories allowed by quantum physics are nonlocal and contextual ones. We give simple examples of these no-go theorems and indicate some interesting problems arising from them for the field of quantization.
\end{abstract}

| Quantization | Groenewold van-Hove theorem | entanglement | contextuality |

\section{Introduction}

In describing quantum physics, one usually faces two obstacles namely the mathematical framework on which the theory is built upon and the realistic interpretation of the ensued theory. It is of course the latter that captures the imagination of many including the laymen. The main problem is being the irreconciliable differences between the classical world and that of the quantum. Progress over the 100 years of quantum has led one to adopt either the extreme position of either [1]

- Quantum theory is fundamental and universal while classical world has only perspectival existence; or

- Quantum theory is only approximate and derived from a more fundamental theory with classical world exists absolutely. 
Our aim here is not really to substantiate any particular position but merely to reemphasize the problem of its mathematical framework that has been most of the time relegated to the background. The importance of mathematical tools in making the philosophical problems more precise is unquestionable, allowing the significant progress made in the last few decades [2], and hence must be made more widely known.

Definite and rigorous as it can be, the mathematics underlying quantum physics is not as rock solid as one thinks; at best it is a set of prescribed mathematical tools attempted to describe most but not all quantum systems. It is thus the intent of this note to highlight some of the open problems associated to quantization. For this purpose, we look into three no-go theorems in quantum theory. First, Groenewold van-Hove theorem [3, 4] is a no-go theorem which is directly related to quantization procedure where the quantization map in usual circumstances could not be extended to the full (polynomial) algebra of observables. The next two no-go theorems are much less related to quantization since they often assume the usual structure of quantum mechanics and how this structure can be made compatible with classical notions through the possibility of hidden variables. They are Bell's theorem [5], which is a no-go theorem for local hidden variables, and Kochen-Specker theorem [6], a nogo theorem for non-contextual hidden variables. We will discuss the necessary ingredients of these theorems with respect to quantization and indicate some possible research directions.

\section{Quantization}

Quantization is essentially a procedure of mapping classical observables i.e. functions of the phase space into quantum ones in the form of self-adjoint operators acting in a suitable Hilbert space. The standard one being the canonical quantization of which one merely looks for operators for conjugate pairs of observables that satisfy the canonical commutation relation (CCR)

$$
\left[\hat{q}^{i}, \hat{p}_{j}\right]=i \hbar \delta_{j}^{i} \hat{1}
$$

It was quickly recognised [7] that similar Lie algebraic structure exists at the classical level (using Poisson bracket $\{\bullet, \bullet\})$ and later the quantization procedure $Q$ is codified as the Dirac problem [8]:

(Q1) $Q\left(f_{1}+f_{2}\right)=Q\left(f_{1}\right)+Q\left(f_{2}\right)$;

(Q2) $Q(a f)=a Q(f), a \in \mathrm{R}$;

(Q3) $Q\left(\left\{f_{1}, f_{2}\right\}\right)=(1 / i \hbar)\left[Q\left(f_{1}\right), Q\left(f_{2}\right)\right]$;

(Q4) $Q(1)=\hat{1}$;

(Q5) $Q\left(q^{i}\right), Q\left(p_{j}\right)$ are unitary equivalent to multiplication operator $q^{j}$ and $(1 / i \hbar)\left(\partial / \partial q^{j}\right)$.

The problem of quantization grew complicated as one goes beyond standard quantum mechanics on vector spaces $\mathrm{R}^{n}$ and the ultimate problem of quantization seems to be quantizing gravity, which is highly nonlinear. On nonlinear spaces, one normally has to take into account the underlying geometry of the system to properly construct a well-defined quantum theory. One such procedure is the geometric quantization [8,9]. The procedure itself begins by finding a prequantization map (involving line bundles) that works on the whole phase space $S$ to ease the treatment of the Dirac problem and later cuts it into half (polarization) to get the operators effectively acting only on the 'configuration space' $M$ (Note that the quantization can also apply to phase spaces with no obvious configuration half). 
A lesser-known quantization method that keeps close to earlier notions of quantization but yet maintains the geometric ingredient is that of Isham's group-theoretic quantization programme [10]. Here, instead one restricts to phase spaces that are cotangent bundles $S=T^{*} M$ of the configuration spaces. The objects sought are canonical groups that describe the global symmetries of the phase space and they are of the form (usually minimal) subgroups of $\operatorname{Diff}(M) \ltimes C^{\infty}(M, \mathrm{R})$ where $\ltimes$ denotes semidirect product. One then simply finds irreducible unitary representations of the canonical group and their generators will furnish the needed quantum observables. Of particular note here, due to the semidirect product structure of the canonical group, Mackey's powerful technique of induced representation theory can be employed [11]. Inequivalent irreducible unitary representations thus pose as different quantizations of the same system. In contrast with geometric quantization, the representations (and hence the operators) need not be on the configuration space and hence flexible enough to give many other quantum systems. This very strength of the programme turns out to be its weakness too since the representations obtained can be very rich to be given a physical interpretation.

For completeness, we ought to mention that there are other variants of the quantization procedure with different starting points and emphasis (see [12]). Most notable to mention here due to their totally different origins and also frequent use in recent problems are Feynman's path integral approach $[13,14]$ and deformation quantization $[15,16]$. The path integral quantization kept close to the tradition of variational problem and 'sums' over all paths of the particle or all field configurations in its formulation (hence the name). This technique is very much in use modern theories of high-energy physics. Deformation quantization as the name implies, deforms the multiplicative structure of observables. This technique has ramifications in areas like quantum groups [17] and $\mathrm{C}^{*}$-algebra unifying framework for quantization [18].

\section{Groenwold-van Hove Theorem}

In the quantization process above, it is often thought that one needs only to generate the map for a few basic observables and other (derived) observables will have their operator status automatically attained. The first problem is of course associated operator ordering ambiguities such as for the combination $q^{i} p_{j}$ whose operator could be $\hat{q}^{i} \hat{p}_{j}$ or $\hat{p}_{j} \hat{q}^{i}$. The text-book solution is of course symmetrising between the two orderings but this supplemented rule however causes problem. As one proceeds to built operator for higher order combinations, one can no longer keep this rule consistent with the suggested map of the Dirac problem. Consider the Poisson bracket identity

$$
\frac{1}{9}\left\{q^{3}, p^{3}\right\}=q^{2} p^{2}=\frac{1}{3}\left\{q^{2} p, p^{2} q\right\}
$$

The left hand side of (2) on quantization gives

$$
\frac{1}{9}\left[Q\left(q^{3}\right), Q\left(p^{3}\right)\right]=Q(q)^{2} Q(p)^{2}-2 i \hbar Q(q) Q(p)-\frac{2}{3} \hbar^{2} I
$$

while its right hand side gives

$$
\frac{1}{3}\left[Q\left(q^{2} p\right), Q\left(p^{2} q\right)\right]=Q(q)^{2} Q(p)^{2}-2 i \hbar Q(q) Q(p)-\frac{1}{3} \hbar^{2} I
$$

This shows that one could not consistently quantize observables beyond quadratic combinations of $q$ 's and $p$ 's! This fragility should come as a shock to those who blindly use the quantization map and this is the basic result of 
the no-go theorem of Groenewold-van Hove. Fortunately in most standard examples in quantum mechanics, such problematic combinations are avoided but then it simply postpones the question of what can we actually consistently quantize.

This theorem was successfully extended to nonlinear phase spaces by Gotay and his co-workers [19-22]. We would like to particular note the case of the two-sphere phase space, $S=S^{2}$ [20], due to our interest in describing qubit geometry. The classical basic observables are taken to be the angular momenta $\left\{S_{1}, S_{2}, S_{3}\right\}$ where they are subjected to the constraint

$$
S_{1}^{2}+S_{2}^{2}+S_{3}^{2}=s^{2}
$$

with $s$ as the angular momentum magnitude. The Poisson bracket for this phase space is given by

$$
\{f, g\}=-\sum_{i, j, k=1}^{3} \varepsilon_{i j k} S_{i} \frac{\partial f}{\partial S_{j}} \frac{\partial g}{\partial S_{k}}
$$

One can verify that (6) reproduces the $\mathrm{SU}(2)$-like algebra

$$
\left\{S_{i}, S_{j}\right\}=-\sum_{k=1}^{3} \varepsilon_{i j k} S_{k}
$$

Analogous to CCR, the quantum relation corresponding to (7) should now read

$$
\left[Q\left(S_{i}\right), Q\left(S_{j}\right)\right]=i \hbar \sum_{k=1}^{3} \varepsilon_{i j k} Q\left(S_{k}\right)
$$

while for relation (5) is

$$
Q\left(\underline{S}^{2}\right)=s^{2} \hat{1}
$$

By theory of angular momenta, one would have expect that

$$
\sum_{i=1}^{3} Q\left(S_{i}\right)^{2}=\hbar^{2} j(j+1) \hat{1}
$$

So let's suppose that the quantization map is given by

$$
Q\left(S_{i}^{2}\right)=a Q\left(S_{i}\right)^{2}+c \hat{1}
$$

and accordingly we can verify that

$$
Q\left(S_{i} S_{j}\right)=\frac{a}{2}\left(Q\left(S_{i}\right) Q\left(S_{j}\right)+Q\left(S_{j}\right) Q\left(S_{i}\right)\right) .
$$

Using this map, one again encounters problem as in the earlier case. Consider 


$$
s^{2} S_{3}=\left\{S_{1}^{2}-S_{2}^{2}, S_{1} S_{2}\right\}-\left\{S_{2} S_{3}, S_{3} S_{1}\right\},
$$

which gives

$$
s^{2}=a^{2} \hbar^{2}\left(j(j+1)-\frac{3}{4}\right)
$$

Another relation is

$$
2 s^{2} S_{2} S_{3}=\left\{S_{2}^{2},\left\{S_{1} S_{2}, S_{1} S_{3}\right\}\right\}-\frac{3}{4}\left\{S_{1}^{2},\left\{S_{1}^{2}, S_{2} S_{3}\right\}\right\}
$$

which gives

$$
s^{2}=a^{2} \hbar^{2}\left(j(j+1)-\frac{9}{4}\right)
$$

Thus, the inconsistency shows that the quantization map given by (11) is not possible; we are only left with quantization map of monomials $S_{i}$ ! This negative result however has its virtue in the sense of restricting the kind of quantum angular momenta observable we can use i.e. the components plus the squared magnitude (which is actually proportional to the identity) and nothing else.

At this juncture, it is tempting to generalize the obstruction to be universal but alas, a counterexample was found by Gotay (resurging the initial interest of the theorem) for the case of $S=T^{2}$ [23], and another instance for $S=T^{*} \mathrm{R}_{+}[22]$. The case of the two-torus is particularly interesting since the assumed phase space is compact but the spectrum obtained is unbounded. This can however be understood if we reinterpret the problem [24] to be on $S=T^{*} T^{2}$ (hence no longer compact) where the symplectic form given in [23] is supplemented by the standard one for the cotangent bundle. The quantization is made possible using group-theoretic quantization [25] where the canonical (sub-)group acting on the configuration space is found to be similar to the Heisenberg-Weyl group and the quantum map in [22] can be realized as one of its inequivalent representation.

\section{Bell's Theorem}

The simplest setting to discuss Bell's theorem is to work with a composite spin-0 state given by what is now known as (one of) the Bell state:

$$
|\Psi\rangle=\frac{1}{\sqrt{2}}(|\uparrow\rangle|\downarrow\rangle-|\downarrow\rangle|\uparrow\rangle)
$$

where $|\uparrow\rangle,|\downarrow\rangle$ are correspondingly the up and down spin- $\frac{1}{2}$ eigenstates. The particles that compose the composite state are allowed to travel far away from each other and subsequent separate measurements $A$ and $B$ can be made onto the individual subsystems. Throwing in further degree of freedom of choice of orientation of measuring the spins along direction $\underline{a}$ at $A$ and $\underline{b}$ at $B$, the correlation between the two measurements in quantum mechanics is given by 


$$
C(\underline{a}, \underline{b})_{\Psi}=\left\langle\Psi\left|\left(\sigma_{1} \cdot \underline{a}\right)\left(\sigma_{2} \cdot \underline{b}\right)\right| \Psi\right\rangle=-\underline{a} \cdot \underline{b} .
$$

If instead, one believes there are local hidden variables $\lambda$ operating and influencing the subsequent results of measurements, then its analogous correlation after tracing over the hidden variable is

$$
C(\underline{a}, \underline{b})=\int d \lambda \rho(\lambda) A_{\underline{a}}(\lambda) B_{\underline{b}}(\lambda)
$$

where $\rho$ is some probability distribution and $A_{\underline{a}}(\lambda)= \pm 1=B_{\underline{b}}(\lambda)$. When $\underline{a}$ and $\underline{b}$ are parallel, we obtain the quantum mechanical result $C(\underline{a}, \underline{a})_{\psi}=-1$ for which we can impose $A_{\underline{a}}(\lambda)=-B_{\underline{a}}(\lambda)$ for consistency of their results. Under this supposition, we have

$$
C(\underline{a}, \underline{b})=-\int d \lambda \rho(\lambda) A_{\underline{a}}(\lambda) A_{\underline{b}}(\lambda) .
$$

Given a third possible orientation $\underline{c}$ for the spin measurement, we can now show that the classical correlation must obey the inequality

$$
|C(\underline{a}, \underline{b})-C(\underline{a}, \underline{c})| \leq 1+C(\underline{b}, \underline{c})
$$

which is one of the family of inequalities known as Bell inequalities. One can easily check that the quantum mechanical result violates this inequality simply by putting geometrically $\underline{a} \cdot \underline{b}=\frac{1}{2}=\underline{b} \cdot \underline{c}$ and $\underline{a} \cdot \underline{c}=-\frac{1}{2}$ to give $1>\frac{1}{2}$.

The failure of (18) is attributed to the property of (14) allowing more correlations than classical locality allows and the state structure being not separable $|\Psi\rangle \neq|\psi\rangle_{1} \otimes|\phi\rangle_{2}$ is indicative of this property. It is instructive to see the problem of such entangled state from the point of view of quantization. Earlier we show that discrete (spin) angular momenta states can be obtained from quantizing the sphere. In the case of the (entangled) Bell state, we would require to quantize the product of two spheres. If straightforward quantization is done on the product of the two-spheres with canonical group $\mathrm{SU}(2) \times \mathrm{SU}(2)$, one could only recover the separable states. Thus some nontrivial quantization is needed to realize entanglement.

Another interesting but not unrelated observation is that the spin- $\frac{1}{2}$ states has the geometry of complex projective lines $\mathrm{C} P^{1}$, which is equivalent to the sphere. Composite spin- $\frac{1}{2}$ states however form $\mathrm{C} P^{3}$ and obviously the product $\mathrm{C} P^{1} \times \mathrm{C} P^{1}$ only forms a submanifold of $\mathrm{C} P^{3}$. Such geometrical characterization of separable quantum states and also entangled ones have been well studied in [26] but whether such geometric information can arise from a quantization procedure remains an open problem.

\section{Kochen-Specker Theorem}

Kochen-Specker theorem is essentially a theorem regarding valuation functions of observables i.e. whether one can consistently assign values to observables. The observables considered are necessarily compatible to avoid 
problems associated with uncertainty principle. It is now known that for dimension of Hilbert space greater than two, one could not do this consistently. Let us demonstrate this for the system of two spin- $\frac{1}{2}$ particles again, giving a Hilbert space of dimension four.

Consider the nine observables for the system given in the Table 1 below [27].

Table 1: Six sets (three rows and three columns) of compatible observables.

\begin{tabular}{|l|l|l|}
\hline $1 \otimes \sigma_{z}$ & $\sigma_{z} \otimes 1$ & $\sigma_{z} \otimes \sigma_{z}$ \\
\hline$\sigma_{x} \otimes 1$ & $1 \otimes \sigma_{x}$ & $\sigma_{x} \otimes \sigma_{x}$ \\
\hline$\sigma_{x} \otimes \sigma_{z}$ & $\sigma_{z} \otimes \sigma_{x}$ & $\sigma_{y} \otimes \sigma_{y}$ \\
\hline
\end{tabular}

Note that observables in each column or row are compatible and according to standard understanding of quantum mechanics, they can be simultaneously measured and assigned values. Another property is that the product of observables in each row or column is \pm 1 and can be summarised in Table 2 .

Table 2: Product of compatible observables.

\begin{tabular}{|l|l|}
\hline R1 & $\left(1 \otimes \sigma_{z}\right) \cdot\left(\sigma_{z} \otimes 1\right) \cdot\left(\sigma_{z} \otimes \sigma_{z}\right)=1 \otimes 1$ \\
\hline R2 & $\left(\sigma_{x} \otimes 1\right) \cdot\left(1 \otimes \sigma_{x}\right) \cdot\left(\sigma_{x} \otimes \sigma_{x}\right)=1 \otimes 1$ \\
\hline R3 & $\left(\sigma_{x} \otimes \sigma_{z}\right) \cdot\left(\sigma_{z} \otimes \sigma_{x}\right) \cdot\left(\sigma_{y} \otimes \sigma_{y}\right)=1 \otimes 1$ \\
\hline C1 & $\left(1 \otimes \sigma_{z}\right) \cdot\left(\sigma_{x} \otimes 1\right) \cdot\left(\sigma_{x} \otimes \sigma_{z}\right)=1 \otimes 1$ \\
\hline C2 & $\left(\sigma_{z} \otimes 1\right) \cdot\left(1 \otimes \sigma_{x}\right) \cdot\left(\sigma_{z} \otimes \sigma_{x}\right)=1 \otimes 1$ \\
\hline C3 & $\left(\sigma_{z} \otimes \sigma_{z}\right) \cdot\left(\sigma_{x} \otimes \sigma_{x}\right) \cdot\left(\sigma_{y} \otimes \sigma_{y}\right)=-1 \otimes 1$ \\
\hline
\end{tabular}

Let us now consider that the individual spins can have definite values and we denote them by $m_{1 z}, m_{z 1}, m_{z z}$, $m_{x 1}, m_{1 x}, m_{x x}, m_{x z}, m_{z x}$ and $m_{y y}$. Table 2 can now be rewritten as Table 3 below. 


\begin{tabular}{|l|l|}
\hline R1 & $m_{1 z} m_{z 1} m_{z z}=1$ \\
\hline R2 & $m_{x 1} m_{1 x} m_{x x}=1$ \\
\hline R3 & $m_{x z} m_{z x} m_{y y}=1$ \\
\hline C1 & $m_{1 z} m_{x 1} m_{x z}=1$ \\
\hline C2 & $m_{z 1} m_{1 x} m_{z x}=1$ \\
\hline C3 & $m_{z z} m_{x x} m_{y y}=-1$ \\
\hline
\end{tabular}

Table 3: Definite (symbolical) value assignments of products.

Note that each $m$ appears twice and there are even number of pairs (six). Thus their product should be equal regardless of \pm 1 values taken by the individual pair. However there is a -1 in the last product of the table above and hence the inconsistency. Note that this inconsistency arises from our insistence that the values are given the same value irrespective of context i.e. noncontextual value assignment. If this is weakened, we can have the following consistent valuation in Table 4

Table 4: Contextual value assignment of products

\begin{tabular}{|l|l|}
\hline R1 & $(1)(1)(1)=1$ \\
\hline R2 & $(1)(1)(1)=1$ \\
\hline R3 & $(1)(1)(1)=1$ \\
\hline C1 & $(-1)(-1)(1)=1$ \\
\hline C2 & $(-1)(-1)(1)=1$ \\
\hline C3 & $(1)(1)(-1)=-1$ \\
\hline
\end{tabular}

Except the black color font, the values with the same color correspond to the same pair of $m$ and are now to be assigned values differently, for example $1 \otimes \sigma_{z}$ has value 1 in R1 but has value -1 in $\mathrm{C} 1$. This time, we need to realize that the consistency is due to the contextuality.

How can we relate this with quantization? Note that the value assignments are essentially from the eigenvalues of the Pauli spin operators. These operators again need to be realized from the quantization of the product of two spheres we alluded earlier in the section before. It is not clear however how can there be an obstruction to noncontextual assignment of eigenvalues but in a preliminary work reported elsewhere [28], we found that the 
eigenvectors of the spin operators can be realized as fixed antipodal points of $\mathrm{C} P^{1}$ under the action of the operators. The case of the product $\mathrm{C} P^{1} \times \mathrm{C} P^{1} \subset \mathrm{C} P^{3}$ may or may not exhibit analogous behaviour due to possible nontrivial group action arising from nonstandard quantization mentioned earlier. We hope to investigate further on this.

\section{Conclusion and Outlook}

We would like to sum up that the mathematical framework of quantum physics is very much open in the context of no-go theorems. In the case of Groenewold-van Hove theorem, it is still an open question as to what extent full quantization can be done and what precisely characterizes the obstruction.

For Bell's and Kochen-Specker theorem, they are now heavily researched by researchers in quantum information and the progress may be used as an input to understand quantization better, particularly in drawing limitations and excesses of the various quantization programmes. Particularly in our case, we would like to see how this governs the choice of canonical group in the group-theoretic quantization scheme. The final goal, of course, is that the progress may also lead to the understanding of the true nature of the quantum itself.

\section{Acknowledgements}

The authors acknowledge UPM and the Ministry of Higher Education Malaysia for the financial support through Fundamental grant 01-20-03-106J and Institute of Advanced Technology, UPM where the research is originally carried out. HZ would like to thank the organizers of AFSS 2006 for which this present article is written and Ibnu Sina Institute, Universiti Teknologi Malaysia for their warm hospitality.

\section{References}

[1] N.P. Landsman, "Between classical and quantum", (arXiv: quant-ph/0506082 v2) to appear in Handbook of Philosophy of Science Vol. 2: Philosophy of Physics, (eds.) J. Earman \& J. Butterfield, Elsevier.

[2] G. Auletta, Foundations and Interpretation of Quantum Mechanics: In the Light of a Critical-Historical Analysis of the Problems and of a Synthesis of the Results, Rev. Edition, World Scientific, Singapore, 2001.

[3] H.J. Groenewold, Physica 12, (1946) 405.

[4] L. van Hove, Proc. Roy. Acad. Sci. Belgium 26, (1951) 1.

[5] J.S. Bell, Physica 1, (1964) 195.

[6] S. Kochen \& E. Specker, J. Math. Mech. 17, (1967) 59-87.

[7] P.A.M. Dirac, The Principles of Quantum Mechanics, Oxford University Press, London, 1958.

[8] N.E. Hurt, Geometric Quantization in Action, D. Reidel, Dordrecht, 1983.

[9] N.M.J. Woodhouse, Geometric Quantization, $2^{\text {nd }}$ Edition, Clarendon Press, Oxford, 1992.

[10] C.J. Isham, "Topological and global aspects of quantum theory" in Relativity, Groups and Topology II, (eds.) B.S. deWitt \& R. Stora, North-Holland, Amsterdam, 1984.

[11] G.W. Mackey, Unitary Group Representations in Physics, Probability and Number Theory, Benjamin/Cummings, Reading, 1978.

[12] S.T. Ali \& M. Englis, Rev. Math. Phys. 17, (2005) 391.

[13] R.P. Feynman \& A.R. Hibbs, Quantum Mechanics and Path Integrals, McGraw Hill, New York, 1965. 
[14] J. Glimm \& A. Jaffe, Quantum Physics: A Functional Integral Point of View, Springer-Verlag, New York, 1987.

[15] F. Bayen, M. Flato, C. Fronsdal, A. Lichnerowicz \& D. Sternheimer, Ann. Phys. 110, (1978) 61, 111.

[16] M.A. Rieffel, Comm. Math. Phys. 122, (1989) 531.

[17] G. Lusztig, Introduction to Quantum Groups, Birkhauser, Boston, 1993.

[18] N.P. Landsman, Mathematical Topics Between Classical and Quantum Mechanics, Springer-Verlag, Berlin, 1998.

[19] M.J. Gotay \& H. Grundling, Rep. Math. Phys. 40, (1997) 107.

[20] M.J. Gotay, H. Grundling \& C.A. Hurst, Trans. Amer. Math. Soc. 348, (1995) 1579.

[21] M.J. Gotay, H. Grundling \& G.T. Tuynman, J. Nonlinear Sci. 6 (1996) 469.

[22] M.J. Gotay, “Obstructions to Quantization”, (arXiv: math-ph/9809011).

[23] M.J. Gotay, "On full quantization of the torus" in Quantization, Coherent States and Complex Structures, (eds.) J.-P. Antoine et al., Plenum, New York, 1995.

[24] H. Zainuddin, "Groenwold-van Hove Problem and Group-Theoretic Quantization of Hall Systems on Torus", in Prosiding Persidangan Fizik Kebangsan, PERFIK 2000, 4-5 September 2000, Port Dickson, Universiti Kebangsaan Malaysia, 2000.

[25] H. Zainuddin, Phys. Rev. D 40, (1989) 636.

[26] M.M. Sinolecka, K. Zyczkowski \& M. Kus, Acta Phys. Pol. B 33, (2002) 2081.

[27] Toh Sing Poh \& H. Zainuddin, "Understanding Basic Idea of Contextuality Through the Quantum Mechanical Interpretation of Schutte's Tautology" to appear in Proc. of ITMA S\&T 2005, ITMA, UPM, 2005.

[28] H. Zainuddin, M. Zainy, J. Hassan, F.P. Zen, M.Y. Sulaiman \& Z.A. Hassan, "Qubit Geometry, Phase Spaces and Quantization" to appear in Proceedings of Conference on Advances in Theoretical Sciences, International Advanced Technology Congress 2005 (CD Proceedings), 6-8 Dec, 2005, IOI Marriott Hotel, Putrajaya 